\title{
15. COCCOLITH STRATIGRAPHY OF MANIHIKI PLATEAU, CENTRAL PACIFIC, DEEP SEA DRILLING PROJECT, SITE 317
}

\author{
David Bukry, United States Geological Survey, La Jolla, California
}

\section{INTRODUCTION}

The conclusion of coring at Manihiki Plateau Site 317, Leg 33, by Glomar Challenger in December 1973 completed a program of the Deep Sea Drilling Project to determine the biostratigraphic history of major submarine plateaus in the Pacific Ocean. Because of their relatively shallow depth, plateaus provide optimum settings for preserving nearly continuous records of planktonic fossils. Major plateaus cored in the Pacific include, from north to south (Figure 1): Hess Rise (DSDP Site 310); Shatsky Rise (DSDP Sites 47, 305, and 306): Magellan Rise (DSDP Site 167); Ontong-Java Plateau (DSDP Sites 64 and 289); Manihiki Plateau (DSDP Site 317): and Lord Howe Rise (DSDP Sites 207 and 208). The initial results of a coccolith study of Manihiki plateau cores are presented here.

Coccolith zonation for the Cenozoic and Mesozoic (Figures 2 and 3 ) is based on the biostratigraphic zonation of Thierstein (1973), Roth (1973), and Bukry (1973, 1975b). A new coccolith species, Discoaster tristellifer, is described and illustrated.

\section{SUMMARY OF COCCOLITH STRATIGRAPHY}

Approximately one coccolith sample per core $(9.5 \mathrm{~m})$ was examined through the 896-meter cored sediment section at Site 317 , lat $11^{\circ} 00.09^{\prime} \mathrm{S}$, long $162^{\circ} 15.78^{\prime} \mathrm{W}$, depth 2622 meters. Drilling disturbance of sediment is reported to be moderate to high (especially in the top section of cores) through most of the cored section of Hole 317B from 0 to 425 meters. Drilling disturbance is not significant in the deeper cores of Hole 317A (402 to $896 \mathrm{~m})$, being more indurated limestone and volcanogenic sandstone. An uncored interval (425 to $554 \mathrm{~m}$ ) separates a Cenozoic section (Pleistocene to early Eocene) from a Mesozoic section (Maestrichtian to Albian or Aptian). Due to poor core recovery, only one sample was available from the lower Eocene section.

The warm-water aspect of Pliocene coccolith assemblages (Cores 2 to 6 ) is indicated by the great abundance of Discoaster brouweri relative to D. surculus and the presence of D. blackstockae among the discoasters, the great abundance of Ceratolithus regosus and Sphenolithus neoabies, the presence of Angulolithina arca and Hayaster perplexus, and the high diversity and abundance of species of Scyphosphaera. A comparison of the relative abundance of the cool-water species $\mathrm{Coc}$ colithus pelagicus with the warm-water genus Discoaster for the upper Pliocene at Site 317 indicates that slightly cooler conditions existed than at tropical plateau sites farther west in the Pacific (Figure 4).

A consistent abundance of species of Discoaster and Sphenolithus through the Cenozoic section at Site 317 implies a persistent relatively warm-water regime. In particular, middle and upper Eocene assemblages contain common Coccolithus formosus, but only a few Chiasmolithus, and no Isthmolithus, whereas Discoaster and Sphenolithus are abundant.

Except for Core 3A (Micula mura Zone) and Core 8A (Eiffellithus turriseiffeli Zone), Cretaceous coccolith assemblages are limited to a few species dominated by Watznaueria barnesae. The overlapping ranges of rare Vagalapilla matalosa and Watznaueria britannica suggest a probable Aptian or Albian age for the deeper cores. In the middle Eocene Reticulofenestra umbilica Zone, the stratigraphic distinction between the Discoaster bifax Subzone and the Discoaster saipanensis Subzone at Site 317 is made by the auxiliary criterion of the evolution of Sphenolithus obtusus populations of the $D$. saipanensis Subzone from the $S$. furcatolithoides population of the D. bifax Subzone. This succession, like others in the genus Sphenolithus, is a useful stratigraphic guide in warm-water areas.

A stratigraphically disjunct occurrence of Discoaster druggii in the Triquetrorhabdulus carinatus Zone assemblages (Figure 5), previously noted in other low-latitude sites (Bukry, in press) indicates a need for reevaluation of coccolith zonal units near the Oligocene-Miocene boundary. The distribution of Discoaster druggii has two distinct abundance peaks in lower Miocene or upper Oligocene sections at Site 317 in the Pacific Ocean and Site 18 in the Atlantic Ocean. A similar stratigraphic occurrence, though less disjunct, perhaps due to higher sedimentation rates, is noted at Site 238 in the Indian Ocean and Site 289 in the Pacific. When first observed, this disjunct pattern of stratigraphic occurrence was considered a result of drilling disturbance of the sediment-slumping of sediment in the drill hole. But the recurrence of the pattern in several oceans and the nonrepeating character in the distribution of some other associated coccoliths make this unlikely. The taxonomic lumping of two similar discoaster species as Discoaster druggii cannot be quickly dismissed, because the skeletal forms of most discoaster specimens in upper Oligocene and lower Miocene pelagic carbonates are obscured by secondary calcite overgrowth. But the range in form of $D$. druggii in the peak populations at Site 317 is practically identical.

Illustration of the form (species character) of Discoaster druggii and other species of these assemblages through several stratigraphic sections-in the manner used for radiolarians by Riedel and Sanfilippo (1971)-will help determine the true nature of the $D$. druggii fluctuations. Preliminary investigation shows considerable variation in the abundance and form of such species as Coccolithus miopelagicus, Cyclicargolithus abisectus, Sphenolithus dissimilis, Triquetrorhabdulus carinatus, and $T$. milowii. 


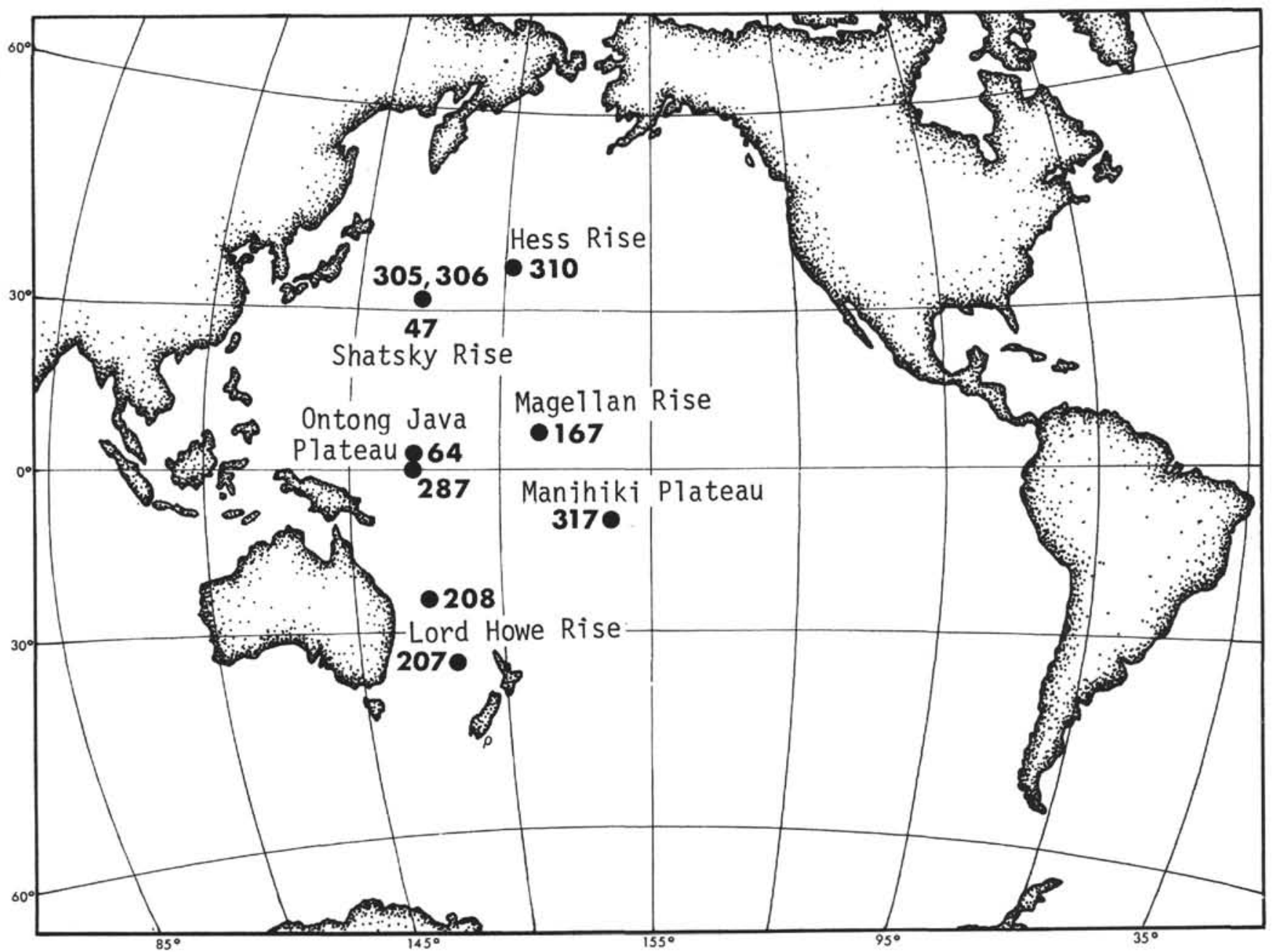

Figure 1. Deep Sea Drilling Project sites at major submarine plateaus in the Pacific Ocean.

\section{COCCOLITHS IN SELECTED SAMPLES, SITE 317}

\section{Upper Pleistocene \\ (Gephyrocapsa oceanica Zone, Emiliania ovata Subzone)}

\section{B-1-1, 75-76 cm (1 m):}

Ceratolithus cristatus (overgrown), Cyclococcolithina leptopora (abundant), Emiliania annula, E. ovata (abundant), Gephyrocapsa oceanica, Hayaster perplexus, Helicopontosphaera kamptneri, $H$. wallichii, Rhabdosphaera claviger, Scyphosphaera apsteinii, S. kamptneri, Syracosphaera histrica.

\section{Upper Pliocene \\ (Discoaster brouweri Zone, Discoaster pentaradiatus Subzone)}

\section{B-2-1, 75-76 cm (8 m):}

Ceratolithus rugosus, Coccolithus pelagicus, Crenalithus doronicoides, Cyclococcolithina macintyrei, Discoaster asymmetricus, D. brouweri, D. pentaradiatus, Discolithina japonica, Emiliania sp. cf. E. ovata, Hayaster perplexus, Helicopontosphaera kamptneri, Rhabdosphaera stylifer, Scyphosphaera pulcherrima, Syracosphaera sp.

\section{Upper Pliocene \\ (Discoaster brouweri Zone, Discoaster tamalis Subzone)}

317B-3-1, 75-76 cm (17 m):

Ceratolithus rugosus, Coccolithus pelagicus (common), Crenalithus doronicoides, Cyclococcolithina macintyrei, Discoaster asymmetricus, D. blackstockae, D. brouweri (abundant), D. decorus, D. pentaradiatus, D. surculus, $D$. tamalis (common), D. variabilis, Discolithina japonica, Emiliania sp. cf. E. ovata, Hayaster perplexus, Helicopontosphaera kamptneri, Oolithotus antillarum, Scyphosphaera pulcherrima.

\section{Lower Pliocene}

(Reticulofenestra pseudoumbilica Zone)

\section{B-4-1, 75-76 cm (27 m):}

Ceratolithus rugosus, Cyclococcolithina leptopora, C. macintyrei, Discoaster asymmetricus, D. blackstockae, $D$. brouweri, D. pentaradiatus, D. surculus, D. tamalis (rare), D. triradiatus, D. variabilis, Reticulofenestra pseudoumbilica, Sphenolithus neoabies.

\section{B-4-4, 100-101 cm (32 m):}

Ceratolithus rugosus (common), Cyclococcolithina leptopora, Discoaster asymmetricus, D. blackstockae, D. 


\begin{tabular}{|c|c|c|c|}
\hline Age & Zone & Subzone & Hole 317B \\
\hline \multirow{5}{*}{ 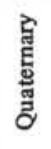 } & \multicolumn{2}{|l|}{ Emiliania huxleyi } & \\
\hline & \multirow{2}{*}{$\begin{array}{l}\text { Gephyrocapsa } \\
\text { oceanica }\end{array}$} & Ceratolithus cristatus & \\
\hline & & Emiliania ovata & $1-1$ \\
\hline & \multirow{2}{*}{$\begin{array}{l}\text { Crenalithus } \\
\text { doronicoides }\end{array}$} & Gephyrocapsa caribbeanica & \\
\hline & & Emiliania annula & \\
\hline \multirow{8}{*}{ 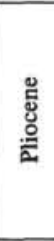 } & \multirow{4}{*}{$\begin{array}{r}\text { Discoaster } \\
\text { brouweri }\end{array}$} & Cyclococcolithina macintyrei & \\
\hline & & Discoaster pentaradiatus & $2-1$ \\
\hline & & Discoaster surculus & \\
\hline & & Discoaster tamalis & 3-1 \\
\hline & \multirow{2}{*}{$\begin{array}{l}\text { Reticulofenestra } \\
\text { pseudoumbilica }\end{array}$} & Discoaster asymmetricus & \\
\hline & & Sphenolithus neoabies & 4-1/4-4 \\
\hline & \multirow{3}{*}{$\begin{array}{l}\text { Ceratolithus } \\
\text { tricorniculatus }\end{array}$} & Ceratolithus rugosus & $5-1 / 6-1$ \\
\hline & & Ceratolithus acutus & \\
\hline \multirow{15}{*}{$\begin{array}{l}\text { ¿ूँ } \\
\frac{\Xi}{\Sigma}\end{array}$} & & Triquetrorhabdulus nugosus & $7-1$ \\
\hline & \multirow{4}{*}{\begin{tabular}{|l|}
$\begin{array}{c}\text { Discoaster } \\
\text { quinqueramus }\end{array}$ \\
Discoaster \\
neohamatus \\
Discoaster
\end{tabular}} & Ceratolithus primus & $8-1 / 11-1$ \\
\hline & & Discoaster berggrenii & \\
\hline & & Discoaster neorectus & \\
\hline & & Discoaster bellus & $12-1$ \\
\hline & Discoaster & Catinaster calyculus & 13-1 \\
\hline & hamatus & Helicopontosphaera kamptneri & \\
\hline & \multicolumn{2}{|l|}{ Catinaster coalitus } & 14-1 \\
\hline & \multirow{2}{*}{\begin{tabular}{|l|} 
Discoaster \\
exilis
\end{tabular}} & Discoaster kugleri & \\
\hline & & Coccolithus miopelagicus & $16-1$ \\
\hline & \multicolumn{2}{|c|}{ Sphenolithus heteromorphus } & $17-1 / 18-1$ \\
\hline & \multirow{2}{*}{\multicolumn{2}{|c|}{$\begin{array}{l}\text { Helicopontosphaera ampliaperta } \\
\text { Sphenolithus belemnos }\end{array}$}} & $19-1$ \\
\hline & & & \\
\hline & \multirow{3}{*}{$\begin{array}{l}\text { Triquetrorhabdulus } \\
\text { carinatus }\end{array}$} & Discoaster druggii & \\
\hline & & Discoaster deflandrei & $20-1 / 25-2$ \\
\hline \multirow{8}{*}{ 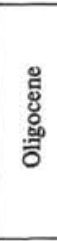 } & & Cyclicargolithus abisectus & \\
\hline & \multirow{2}{*}{$\begin{array}{c}\begin{array}{c}\text { Sphenolithus } \\
\text { ciperoensis }\end{array} \\
\end{array}$} & Dictyococcites bisectus & $26-2 / 28-1$ \\
\hline & & Cyclicargolithus floridanus & $29-1 / 30-1$ \\
\hline & \multicolumn{2}{|c|}{ Sphenolithus distentus } & \\
\hline & \begin{tabular}{|l} 
Sphenolithus predist \\
\end{tabular} & & $31-2 / 34-1$ \\
\hline & & Reticulofenestra hillae & \\
\hline & reticulata & Coccolithus formosus & \\
\hline & & Coccolithus subdistichus & \\
\hline & Discoaster & Isthmolithus recunvus & \\
\hline & barbadiensis & Chiasmolithus oamaruensis & $35-1 / 37-1$ \\
\hline & Reticulofenestra & Discoaster saipanensis & $38-1$ \\
\hline & umbilica & Discoaster bifax & $39-1 / 40-1$ \\
\hline & & Coccolithus staurion & \\
\hline & Nannotritina & Chiasmolithus gigas & \\
\hline & & Discoaster strictus & \\
\hline & Discoaster & Rhabdosphaera inflata & \\
\hline & sublodoensis & Discoasteroides kuepperi & \\
\hline & $\begin{array}{l}\text { Discoaster lodoensis } \\
\end{array}$ & & \\
\hline & Tribrachiatus orthos & & \\
\hline & Discoaster & Discoaster binodosus & $2-1$ \\
\hline & diastypus & Tribrachiatus contortus & Hole 317A \\
\hline & Discoaster & Campylosphaera eodela & \\
\hline & multiradiatus & Chiasmolithus bidens & \\
\hline & Discoaster nobilis & & \\
\hline हूँ & Discoaster mohleri & & \\
\hline 光 & Heliolithus kleinpell & & \\
\hline & Fasciculithus tympa & ormis & \\
\hline & Cruciplacolithus tem & & \\
\hline
\end{tabular}

Figure 2. Cenozoic coccolith zonation of studied core sections from Deep Sea Drilling Project, Site 317.

brouweri, D. pentaradiatus, D. tamalis, D. tristellifer, D. variabilis, Reticulofenestra pseudoumbilica, Scyphosphaera deflandrei, S. globulata, S. kamptneri, S. pulcherrima, $S$. recurvata, Sphenolithus abies, $S$. neoabies.

\section{Lower Pliocene \\ (Ceratolithus tricorniculatus Zone, Ceratolithus rugosus Subzone)}

317B-5-1, 75-76 cm (36 m):

Angulolithina arca, Ceratolithus primus, $C$. rugosus, $C$. tricorniculatus, Crenalithus sp. cf. $C$. doronicoides, Cyclococcolithina leptopora, Discoaster asymmetricus,

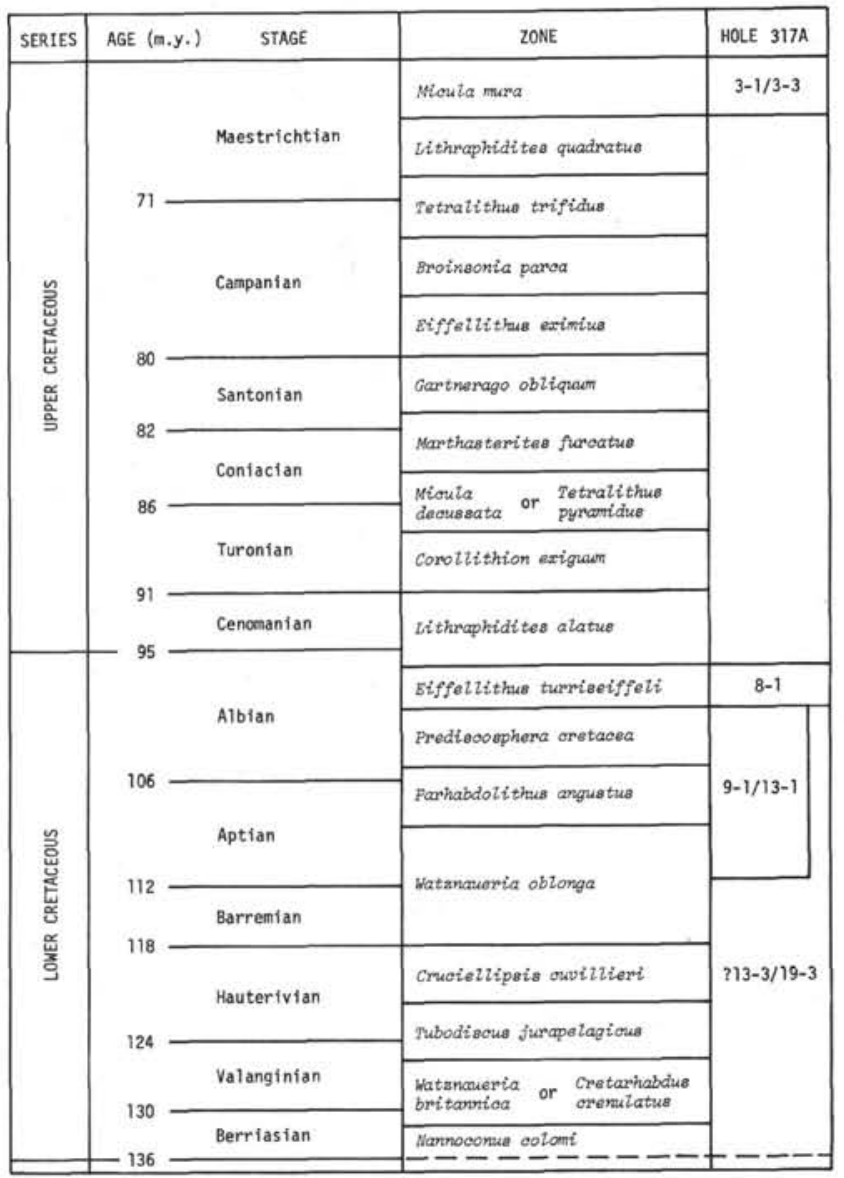

Figure 3. Mesozoic coccolith zonation of cores from Deep Sea Drilling Project, Site 317.

D. blackstockae, D. brouweri, D. pentaradiatus, D. surculus, D. triradiatus, Helicopontosphaera kamptneri, Reticulofenestra pseudoumbilica, Scyphosphaera sp. cf. $S$. conica, S. globulata, S. pulcherrima, Sphenolithus neoabies.

317B-6-1, 75-76 cm (46 m):

Angulolithina arca, Ceratolithus acutus, $C$. armatus, $C$. primus, C. rugosus, Coccolithus pelagicus, Cyclococcolithina macintyrei, Discoaster brouweri, D. pentaradiatus, D. surculus, Helicopontosphaera kamptneri, Reticulofenestra pseudoumbilica, Scyphosphaera apsteinii, $S$. globulosa, $S$. globulata, $S$. intermedia, $S$. kamptneri, $S$. pulcherrima, $S$. recurvata, $S$. spp., Sphenolithus abies.

\section{Upper Miocene}

(Ceratolithus tricorniculatus Zone, Triquetrorhabdulus rugosus Subzone)

317B-7-1, 75-76 cm (55 m):

Ceratolithus primus, Coccolithus pelagicus (abundant), Cyclococcolithina leptopora, Discoaster asymmetricus, $D$. brouweri, $D$. pentaradiatus, D. surculus, D. variabilis, Helicopontosphaera kamptneri, Reticulofenestra pseudoumbilica, Scyphosphaera globulata, $S$. pulcherrima, $S$. recurvata, Sphenolithus abies, Triquetrorhabdulus rugosus (common). 


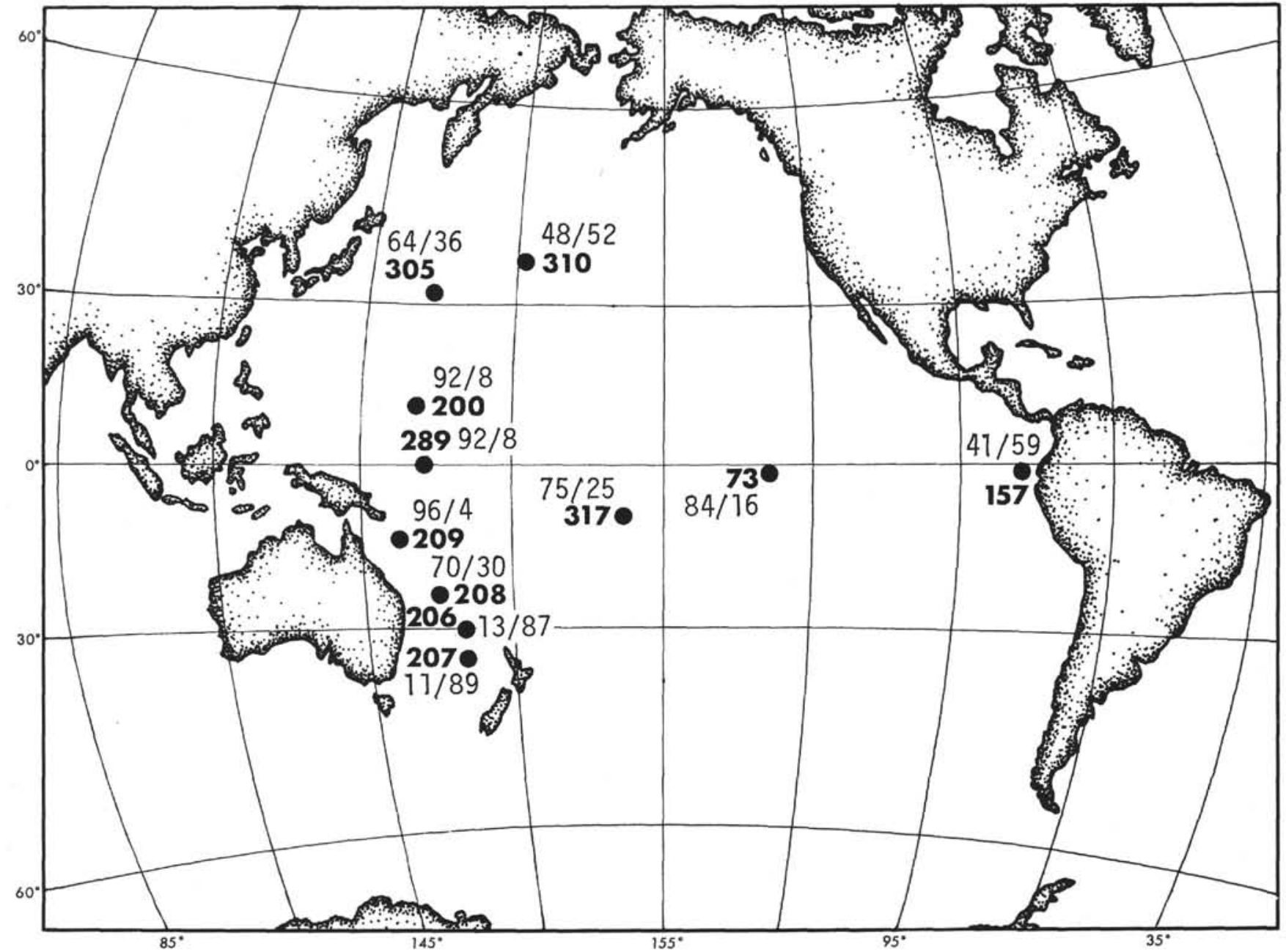

Figure 4. Discoaster/Coccolithus ratio of percentages for the upper Pliocene Discoaster pentaradiatus Subzone at an array of DSDP Sites through the Pacific Ocean. A lowered proportion of Discoaster suggests cooler conditions.

\section{Upper Miocene \\ (Discoaster quinqueramus Zone, Ceratolithus primus Subzone)}

\section{B-8-1, 75-76 cm (64 m):}

Ceratolithus amplificus (common), C. primus, Coccolithus pelagicus, Cyclococcolithina leptopora, C. macintyrei, Discoaster asymmetricus, $D$. sp. cf. D. bellus, $D$. brouweri, D. pentaradiatus, D. quinqueramus, D. surculus, D. variabilis, Helicopontosphaera kamptneri, Reticulofenestra pseudoumbilica, Scyphosphaera pulcherrima, $S$. recurvata, $S$. spp., Sphenolithus abies, Triquetrorhabdulus rugosus.

\section{B-11-1, 95-96 cm (93 m):}

Ceratolithus primus (rare), Coccolithus pelagicus, Cyclococcolithina macintyrei, Discoaster brouweri s. ampl., D. pentaradiatus, D. quinqueramus (rare), D. surculus, Helicopontosphaera kamptneri, Reticulofenestra pseudoumbilica, Scyphosphaera piriformis, S. recurvata, Sphenolithus abies, Triquetrorhabdulus rugosus.

\section{Upper Miocene}

(Discoaster neohamatus Zone)

317B-12-1, 75-76 cm (103 m):

Coccolithus pelagicus, Cyclococcolithina leptopora (abundant), C. macintyrei, Discoaster asymmetricus, D. braarudii, D. brouweri rutellus, D. neohamatus, D. pentaradiatus, D. prepentaradiatus, D. variabilis, Helicopontosphaera kamptneri, Minylitha convallis, Reticulofenestra pseudoumbilica, Scyphosphaera pulcherrima, S. recurvata, Sphenolithus abies, S. neoabies (abundant), Triquetrorhabdulus rugosus.

\section{Middle Miocene}

(Discoaster hamatus Zone, Catinaster calyculus Subzone)

317B-13-1, 75-76 cm (112 m):

Catinaster calyculus, Coccolithus pelagicus, Cyclococcolithina macintyrei, Discoaster bellus, D. hamatus, D. variabilis, Reticulofenestra pseudoumbilica, Scyphosphaera recurvata, Sphenolithus neoabies, Triquetrorhabdulus rugosus (abundant).

Middle Miocene

(Catinaster coalitus Zone)

317B-14-1, 75-76 cm (121 m):

Catinaster sp. cf. C. coalitus, Coccolithus miopelagicus, C. pelagicus, Cyclococcolithina leptopora, C. macintyrei, Discoaster braarudii, D. challengeri, D. moorei, D. sp. cf. D. variabilis, Helicopontosphaera granulata, Reticulofenestra pseudoumbilica, Triquetrorhabdulus rugosus (large). 


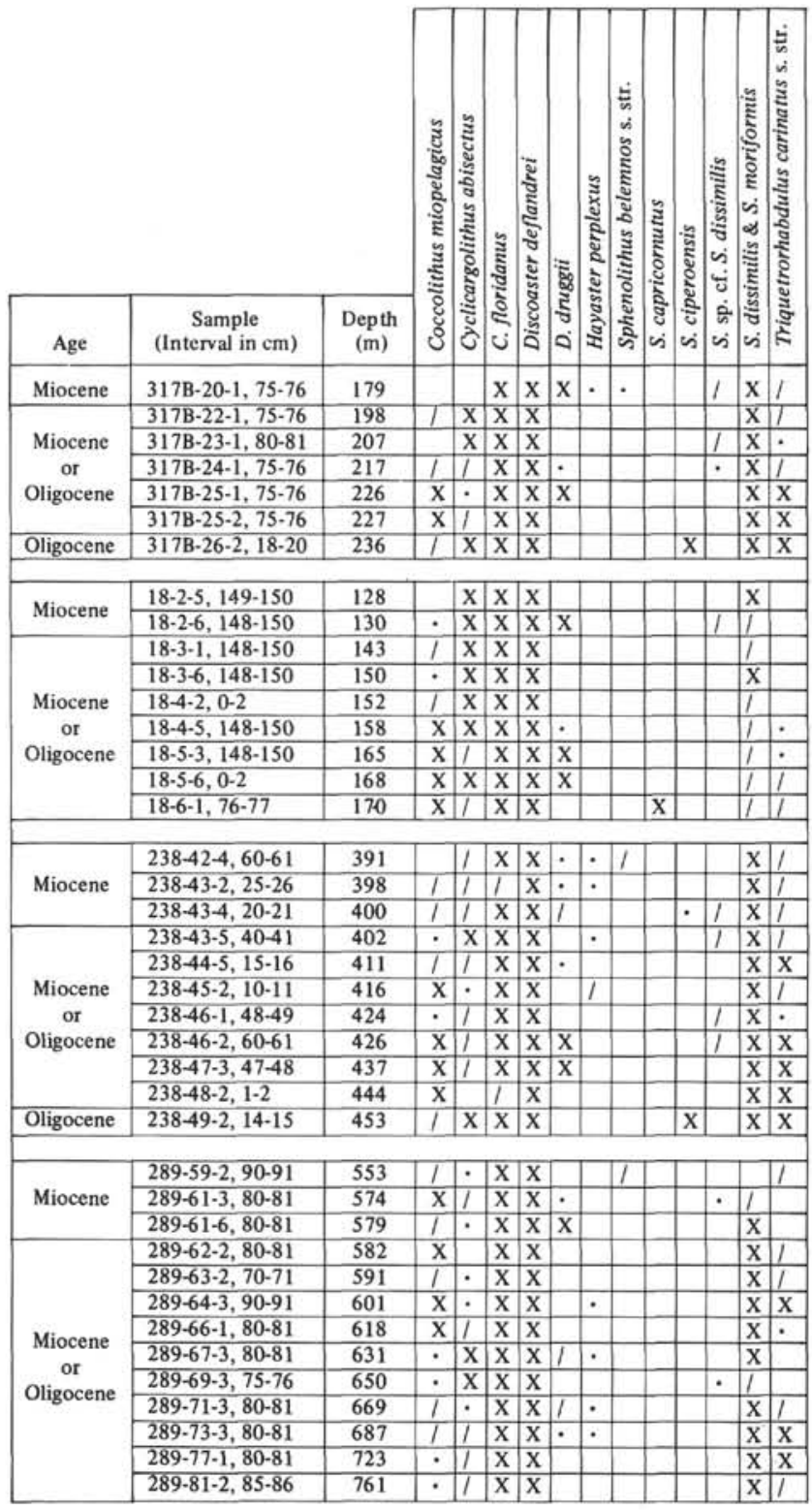

Figure 5. Disjunct stratigraphic occurrence of Discoaster druggii and other species fluctuations in low-latitude sections of DSDP Sites $317,18,238$, and 289. $\mathrm{X}=$ common to abundant; $\mid=$ few; $\cdot=$ rare.

\section{Middle Miocene \\ (Discoaster exilis Zone, Coccolithus miopelagicus Subzone)}

317B-16-1, 75-76 cm (141 m):

Coccolithus miopelagicus, $C$. pelagicus, Coronocyclus nitescens, Cyclicargolithus floridanus, Cyclococcolithina macintyrei, Discoaster sp. cf. D. challengeri, D. sp. cf. $D$. deflandrei, D. sp. cf. D. exilis, Helicopontosphaera granulata, Orthorhabdus serratus (common), Reticulofenestra pseudoumbilica, Sphenolithus neoabies, Triquetrorhabdulus rugosus.

\section{Middle Miocene \\ (Sphenolithus heteromorphus Zone)}

317B-17-1, 103-104 cm (149 m):

Coccolithus miopelagicus, $C$. pelagicus, Coronocyclus nitescens, Cyclicargolithus floridanus, Discoaster deflandrei, D. sp. cf. D. exilis, D. signus, Discolithina segmenta, Helicopontosphaera granulata, H. kamptneri, H. rhomba, Sphenolithus heteromorphus (abundant).

\section{Lower Miocene \\ (Helicopontosphaera ampliaperta Zone)}

317B-19-1, 75-76 cm (169 m):

Coccolithus miopelagicus, C. pelagicus, Coronocyclus nitescens, Cyclicargolithus floridanus, Discoaster deflandrei (abundant), D. druggii, D. sp. cf. D. exilis, Discolithina sp., Hayaster perplexus, Orthorhabdus serratus, Sphenolithus heteromorphus (abundant), S. moriformis.

\section{Lower Miocene}

(Triquetrorhabdulus carinatus Zone)

317B-20-1, 75-76 cm (179 m):

Coccolithus pelagicus (abundant), Coronocyclus nitescens, Cyclicargolithus floridanus, Cyclococcolithina leptopora (rare), Discoaster deflandrei, D. druggii (common), Hayaster perplexus, Orthorhabdus serratus, Sphenolithus dissimilis, S. moriformis, Triquetrorhabdulus carinatus.

317B-22-1, 75-76 cm (198 m):

Coccolithus miopelagicus, C. pelagicus, Coronocyclus nitescens, Cyclicargolithus abisectus, C. floridanus, Discoaster deflandrei, Heliconpontosphaera euphratis, Sphenolithus conicus, $S$. dissimilis, $S$. moriformis (small, abundant), Triquetrorhabdulus carinatus.

317B-23-1, 75-76 cm (207 m):

Coccolithus pelagicus, Coronocyclus nitescens, Cyclicargolithus abisectus, C. floridanus, Discoaster deflandrei, Discolithina segmenta, Helicopontosphaera euphratis, Sphenolithus conicus, S. dissimilis, S. moriformis, Triquetrorhabdulus milowii.

317B-24-1, 75-76 cm (217 m):

Coccolithus miopelagicus, Coronocyclus nitescens, Cyclicargolithus floridanus, Discoaster deflandrei, D. sp. cf. D. druggii (rare), Sphenolithus sp. cf. S. belemnos, $S$. dissimilis, S. moriformis, Triquetrorhabdulus carinatus, T. milowii.

317B-25-1, 75-76 cm (226 m):

Coccolithus eopelagicus, C. miopelagicus, Coronocyclus nitescens, Cyclicargolithus floridanus, Discoaster deflandrei, D. druggii (common), Sphenolithus moriformis, Triquetrorhabdulus carinatus (abundant), $T$. milowii.

317B-25-2, 75-76 cm (227 m):

Coccolithus sp. cf. C. fenestratus, C. miopelagicus, Cyclicargolithus abisectus, C. floridanus, Discoaster de- 
flandrei, Sphenolithus conicus, S. dissimilis, S. moriformis, Triquetrorhabdulus carinatus (abundant).

\section{Upper Oligocene}

(Sphenolithus ciperoensis Zone, Dictyococcites bisectus Subzone)

317B-26-2, 18-20 cm (236 m):

Coccolithus sp. cf. C. fenestratus, C. miopelagicus, $C$. pelagicus, Coronocyclus nitescens, Cyclicargolithus abisectus, C. floridanus, Discoaster deflandrei, Sphenolithus ciperoensis (rare), S. conicus, $S$. dissimilis, $S$. moriformis, Triquetrorhabdulus carinatus (abundant).

317B-29-1, 75-76 cm (264 m):

Coccolithus eopelagicus, $C$. sp. cf. $C$. fenestratus, $C$. miopelagicus, Cyclicargolithus abisectus,, C. floridanus, Discoaster deflandrei, Sphenolithus ciperoensis, S. distentus, $S$. moriformis.

\section{Lower Oligocene \\ (Sphenolithus predistentus Zone)}

\section{B-32-1, 75-76 cm (293 m):}

Coccolithus eopelagicus, $C$. sp. cf. $C$. fenestratus, $C$. pelagicus, Coronocyclus sp., Cyclicargolithus floridanus, Dictyococcites bisectus, D. scrippsae, Discoaster deflandrei, D. tanii, Sphenolithus moriformis (common), Sphenolithus predistentus (long, bifurcate variety abundant), $S$. pseudoradians.

\section{Upper Eocene \\ (Discoaster barbadiensis Zone)}

\section{B-35-1, 75-76 cm (321 m):}

Bramletteius serraculoides (abundant), Coccolithus eopelagicus, C. formosus, C. pelagicus, Cyclicargolithus floridanus, Dictyococcites bisectus (abundant), Dictyococcites scrippsae, Discoaster barbadiensis, D. deflandrei, D. saipanensis, Reticulofenestra umbilica, Sphenolithus moriformis, $S$. predistentus.

\section{B-37-1, 85-86 cm (340 m):}

Bramletteius serraculoides, Ceratolithina? vesca, Coccolithus eopelagicus, C. formosus (abundant), $C$. pelagicus, Cyclolithella? kingii, Dictyococcites bisectus, D. scrippsae (abundant), Discoaster barbadiensis, D. nodifer, D. saipanensis, D. tanii (abundant), Reticulofenestra umbilica, Sphenolithus moriformis (small, abundant), $S$. pseudoradians, $S$. sp. cf. $S$. radians.

\section{Middle Eocene}

(Reticulofenestra umbilica Zone, Discoaster saipanensis Subzone)

\section{B-38-1, 137-138 cm (349 m):}

Bramletteius serraculoides, Campylosphaera dela, Chiasmolithus grandis, C. solitus (rare), Coccolithus eopelagicus, C. formosus, C. pelagicus, Coronocyclus sp., Cyclicargolithus floridanus, Dictyococcites bisectus, $D$. scrippsae (abundant), Discoaster barbadiensis, D. nodifer, D. saipanensis, Helicopontosphaera heezenii, Reticulofenestra umbilica, Sphenolithus obtusus (abundant), $S$. pseudoradians, $S$. spiniger, Triquetrorhabdulus inversus.

\section{Middle Eocene \\ (Reticulofenestra umbilica Zone, Discoaster bifax Subzone)}

317B-39-1, 75-76 cm (359 m):

Bramletteius serraculoides, Campylosphaera dela, Chiasmolithus grandis, C. solitus, C. titus, Coccolithus eopelagicus, C. formosus, Dictyococcites scrippsae (rare), Discoaster barbadiensis, $D$. nodifer, $D$. saipanensis, Helicopontosphaera heezenii, Reticulofenestra samodurovi, $R$. umbilica, Sphenolithus furcatolithoides, $S$. moriformis, $S$. pseudoradians, $S$. spiniger, Triquetrorhabdulus inversus.

\section{B-40-1, 130-131 cm (369 m):}

Bramletteius serraculoides, Campylosphaera dela, Chiasmolithus grandis (common), C. solitus, C. titus, Coccolithus eopelagicus, $C$. formosus (abundant), C. miopelagicus, C. pelagicus, Cyclolithella ? aprica, C.? bramlettei, Discoaster barbadiensis, Helicopontosphaera heezenii, Nannotetrina sp., Reticulofenestra samodurovi, $R$. umbilica, Sphenolithus furcatolithoides (abundant), $S$. pseudoradians (rare), S. spiniger, Striatococcolithus sp., Thoracosphaera prolata, Triquetrorhabdulus inversus.

\section{Lower Eocene \\ (Discoaster diastypus Zone)}

\section{A-2-1, 75-76 cm (555 m):}

Campylosphaera eodela, Chiasmolithus bidens, C. californicus, C. consuetus, C. sp. cf. C. grandis, Coccolithus pelagicus, Cyclolithella? sp., Discoaster barbadiensis, D. diastypus, D. nobilis, D. salisburgensis, Ellipsolithus macellus, Sphenolithus moriformis, $S$. sp. cf. $S$. radians, Toweius sp. cf. T. eminens, Tribrachiatus sp. cf. $T$. orthostylus (thick overgrowth), Zygodiscus adamas, Zygolithus protenus. This sample and the upper part of the core are considered to be displaced by slumping during drilling. Shipboard scientists report a Maestrichtian age for the lower part of the core.

\section{Upper Maestrichtian}

(Micula mura Zone)

317A-3-1, 75-76 cm (565 m):

Arkhangelskiella cymbiformis, Cretarhabdus crenulatus, C. schizobrachiatus, Cribrosphaera ehrenbergii (abundant), Cylindralithus gallicus, C. serratus, Eiffellithus turriseiffeli, Manivitella gronosa, Markalius circumradiatus (of Perch-Nielsen, 1968), Microrhabdulus decoratus, Micula decussata, M. mura, Parhabdolithus sp. cf. P. angustus, Prediscosphaera cretacea, P. lata, Stephanolithion laffitei, Watznaueria barnesae, W. biporta, Zygodiscus sigmoides.

317A-3-3, 75-76 cm (568 m):

Arkhangelskiella cymbiformis, Chiastozygus sp., Cretarhabdus crenulatus, C. schizobrachiatus, Cribrosphaera ehrenbergii, Cylindralithus gallicus, C. serratus, Manivitella gronosa, Markalius circumradiatus (of Perch-Nielsen, 1968), M. inversus, Microrhabdulus decoratus, Micula decussata, M. mura, Parhabdolithus sp. cf. P. angustus, Prediscosphaera cretacea, Watznaueria barnesae, $W$. biporta, Zygodiscus spiralis. 


\section{Albian \\ (Eiffellithus turriseiffeli Zone)}

317A-8-1, 125-126 cm (602 m):

Biscutum testudinarium (abundant), Chiastozygus sp., Cretarhabdus crenulatus, Cribrosphaera primitiva (rare), Eiffellithus turriseiffeli, Lithastrinus floralis, Manivitella pemmatoidea, Parhabdolithus embergeri, Vagalapilla matalosa, Watznaueria barnesae (abundant), W. britannica, W. ovata, Zygodiscus bicrescenticus.

\section{Albian or Aptian}

317A-9-1, $75-76 \mathrm{~cm}$ (612 m):

Vagalapilla matalosa, Watznaueria barnesae (abundant), $W$. biporta, $W$. britannica, $W$. manivitae, $W$. ovata.

$317 \mathrm{~A}-10-1,120-121 \mathrm{~cm}(622 \mathrm{~m})$ :

Cretarhabdus crenulatus, Lithastrinus floralis, Parhabdolithus embergeri, Rhagodiscus asper, Vagalapilla matalosa, V. stradneri, Watznaueria barnesae (abundant), $W$. biporta, W. britannica, Zygodiscus $\mathrm{sp}$.

317A-13-1, 143-144 cm (650 m):

Vagalapilla sp. cf. V. matalosa, Watznaueria barnesae.

\section{Lower Cretaceous}

317A-13-3, 87-88 cm (652 m):

Cretarhabdus crenulatus, Cyclagelosphaera margerelii, Parhabdolithus sp. cf. P. angustus, Rhagodiscus asper, Watznaueria barnesae (abundant), W. sp. cf. $W$. bayackii, W. ovata.

\section{Mesozoic}

317A-19-3, 75-76 cm (719 m):

Watznaueria barnesae (few, poorly preserved).

\section{TAXONOMY}

Discoaster tristellifer n. sp.

(Plate 1, Figures 1-17)

Description: Discoaster tristellifer is typically six rayed and is characterized by star-shaped knobs of different diameter on opposite sides of the central area. The main rays are long and taper from simple points or slightly indented tips toward the central area. The larger of the two central knobs fills most of the central area and has its six points aligned with the discoaster rays. The smaller knob, projecting from the center of the discoaster on the opposite side, has its points aligned between the discoaster rays.
Remarks: Discoaster tristellifer is distinguished from other species by the combination of its long, slender rays and two central-area knobs of different diameter. D. bollii Martini and Bramlette has short, broad, bifurcate rays and knobs of similar diameter. D. bifax Bukry has knobs of different diameter but is distinguished by a rosette ray pattern. D. altus Müller has one large central knob and is distinguished by being short-rayed and flat rather than double knobbed. $D$. quinqueramus Gartner has a large central knob, but only on one side, and is five rayed.

Through-focal series for three specimens of Discoaster tristellifer (Plate 1, Figures 1-7, 9-12, and 14-16) show the distinctly different diameters of the two central-area knobs.

Occurrence: Discoaster tristellifer has a limited occurrence, being presently recorded only in lower Pliocene warm-water assemblages of Site 317 in the Pacific and Site 242 in the Indian Ocean.

Size: 10-20 micrometers diameter; holotype 16 micrometers.

Holotype: USNM 216215 (Plate 1, Figures 1-7).

Paratypes: USNM 216216 to 216220 .

Type locality: Western Indian Ocean, Sample 242-3-1, 60-61 cm $(129 \mathrm{~m})$.

\section{REFERENCES}

Bukry, D., 1973. Low-latitude coccolith biostratigraphic zonation. In Edgars, N.T., Saunders, J.B., et al., Initial Reports of the Deep Sea Drilling Project, Volume 15: Washington (U.S. Government Printing Office), p. 685-703.

, 1975. Coccolith and silicoflagellate stratigraphy, northwestern Pacific Ocean, Deep Sea Drilling Project Leg 32. In Larson, R.L., Moberly, R., et al., Initial Reports of the Deep Sea Drilling Project, Volume 32: Washington (U.S. Government Printing Office), p. 677-702.

in press. Phytoplankton stratigraphy, southwest Pacific, Deep Sea Drilling Project Leg 30. In Andrews, J.E., Packham, G., et al., Initial Reports of the Deep Sea Drilling Project, Volume 30: Washington (U.S. Government Printing Office).

Perch-Nielsen, K., 1968. Der Feinbau und die Klassifikation der Coccolithen aus dem Maastrichtien von Dänemark: Biol. Skr. Danske Vidensk. Selsk., v. 16, p. 1-96.

Riedel, W.R. and Sanfilippo, A., 1971. Cenozoic Radiolaria from the western tropical Pacific, Leg 7. In Winterer, E.L., Riedel, W.R., et al., Initial Reports of the Deep Sea Drilling Project, Volume 7: Washington (U.S. Government Printing Office), p. 1529-1672.

Roth, P.H., 1973. Calcareous nannofossils-Leg 17, Deep Sea Drilling Project. In Winterer, E.L., Ewing, J.I., et al., Initial Reports of the Deep Sea Drilling Project, Volume 17: Washington (U.S. Government Printing Office), p. 695-795.

Thierstein, H.R., 1973. Lower Cretaceous calcareous nannoplankton biostratigraphy: Osterreichische Geol. Bundesanst. Abh., v. 29, p. 1-52. 


\section{PLATE 1}

Discoasters from DSDP Leg 33 and Leg 25

Magnification $2200 \times$; scale bar equals 5 micrometers

Figures 1-17 Discoaster tristellifer $\mathrm{n}$. sp.

1-7. Holotype, USNM 216215, Sample 242-3-1, 60-61 cm (129 m).

8. USNM 216216, Sample 242-3-1, 60-61 cm (129 $\mathrm{m})$.

9-12. USNM 216217, Sample 242-3-1, 60-61 cm $(129 \mathrm{~m})$.

13. USNM 216218, Sample 242-3-1, 60-61 cm $(129 \mathrm{~m})$.

14-16. USNM 216219, Sample 316B-4-4, 100-101 $\mathrm{cm}(32 \mathrm{~m})$.

17. USNM 216220, Sample 317B-4-4, 100-101 cm $(32 \mathrm{~m})$.

Figure 18 Discoaster pansus (Bukry and Percival). Sample 242-3-1, 60-61 cm (129 m).

Figure 19 Discoaster surculus Martini and Bramlette. Sample 242-3-1, 60-61 cm (129 m). 


\section{PLATE 1}
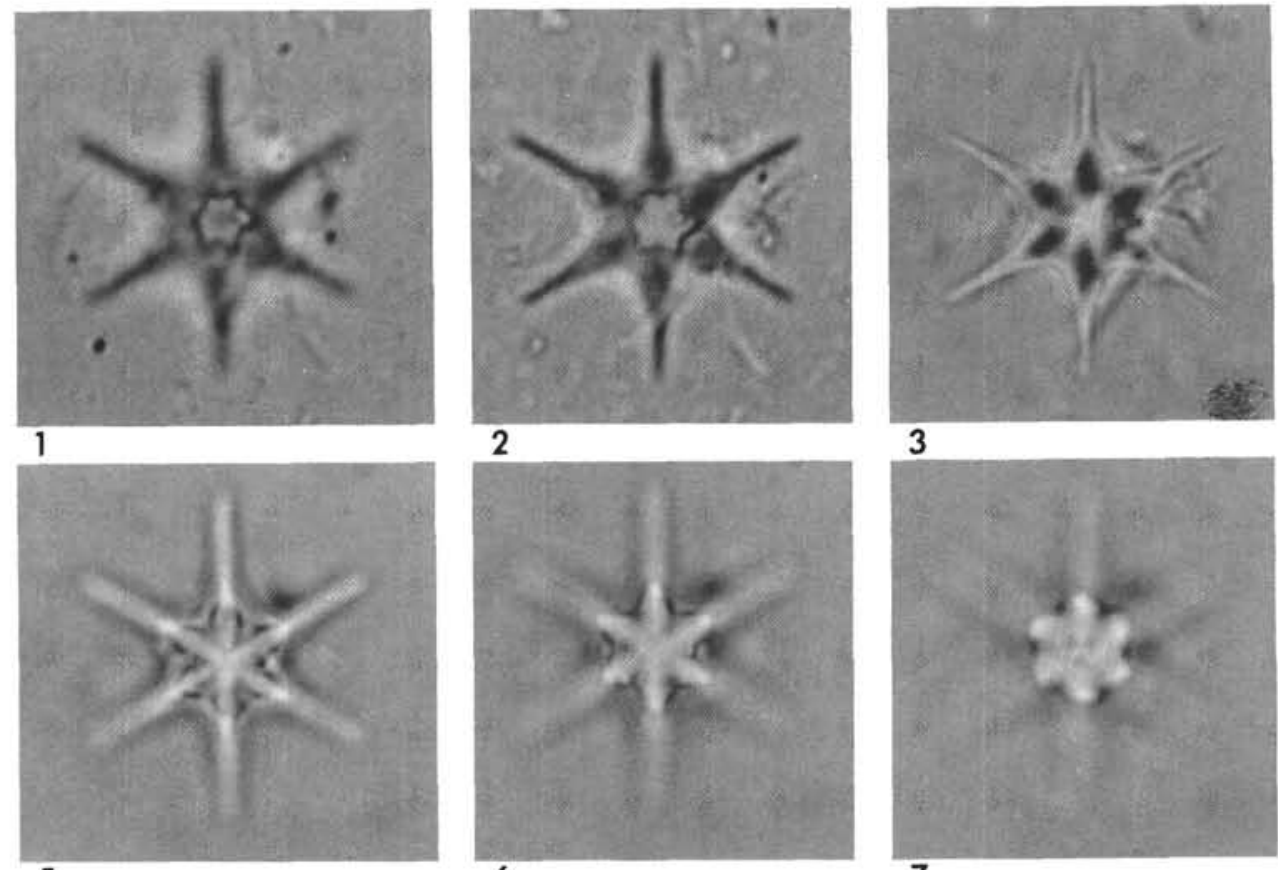

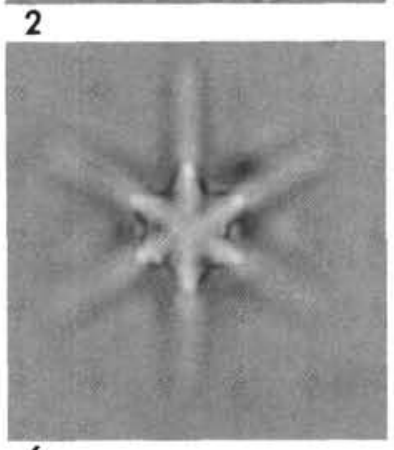

3
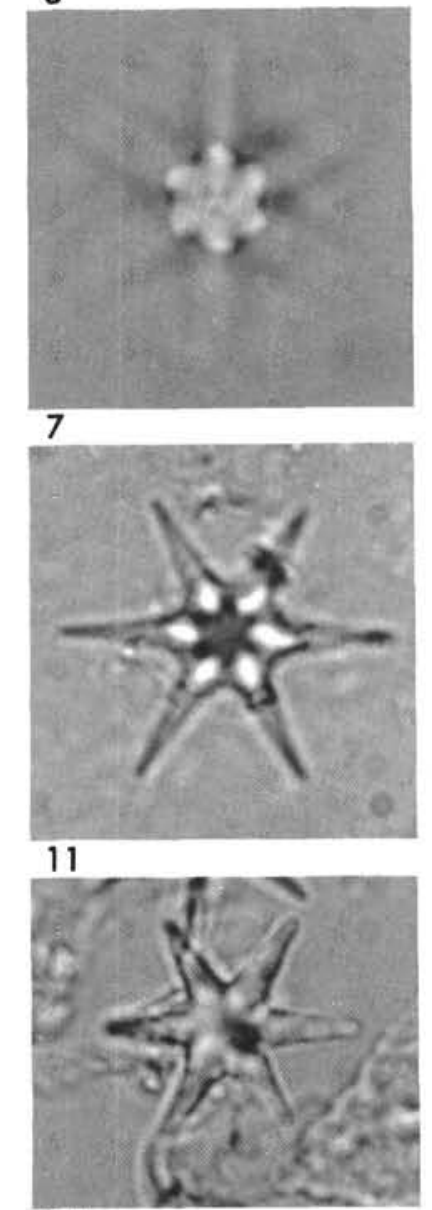

15

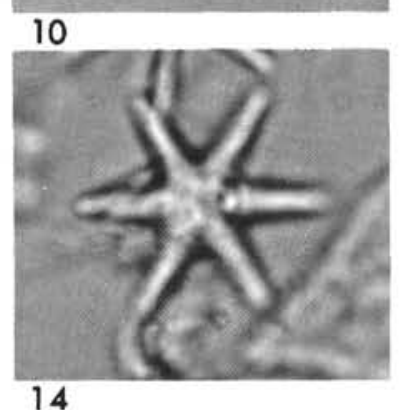

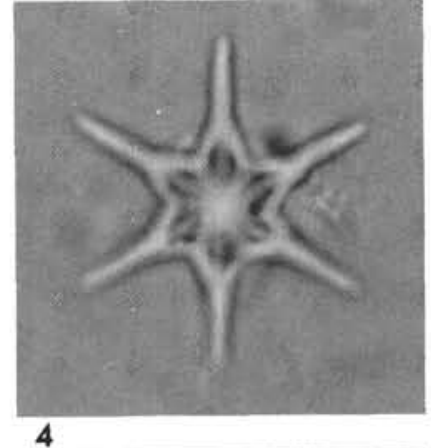

4

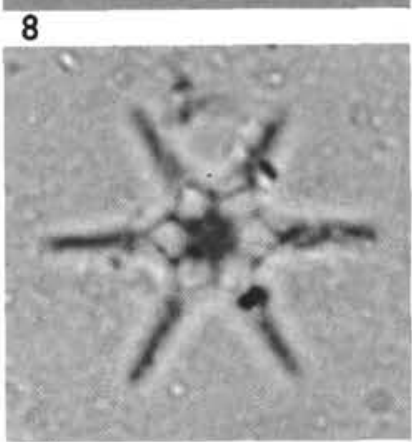

12

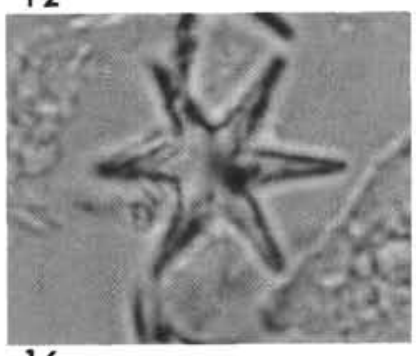

16

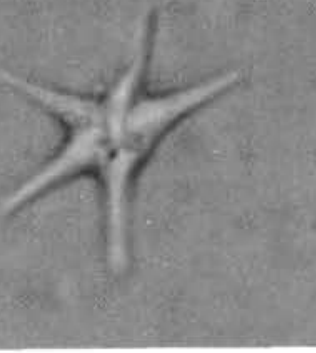

13

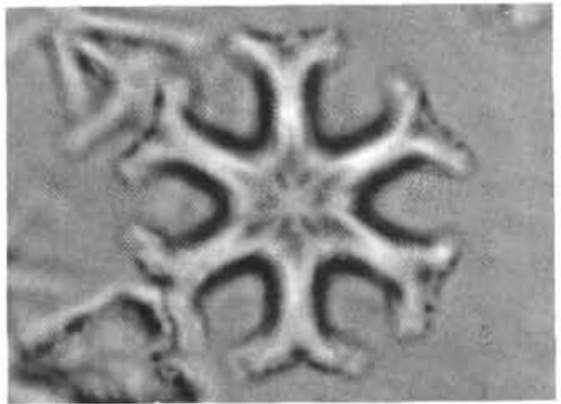

18

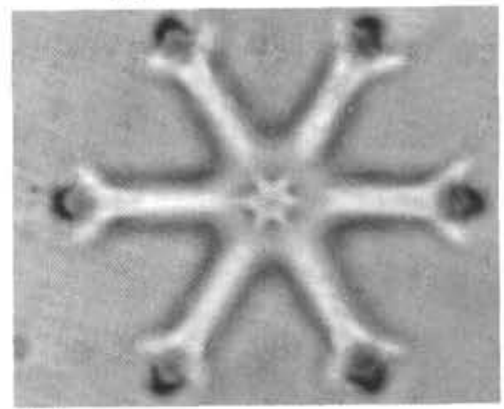

19 